\title{
Phylogeny of immunity
}

Nicholas Cohen

Immunity in Evolution. By John J. Marchalonis. Pp. $\mathrm{Xx}+316$. (Edward Arnold: London, 1977.) £10.

RECENT years have witnessed a surge of quality research and heightened theoretical interest in the plethora of problems posed by the phylogeny of immunity. Within the past two years, this activity has led to the birth of the journal, Developmental and Comparative Immunology, and to the formation of the Division of Comparative Immunology within the construct of the American Society of Zoologists. This activity has also been translated into several reviews, publication of the proceedings of four substantative international symposia, and, as well might be expected, the appearance of three monographs. Dr Marchalonis' labour of love, Immunity in Evolution, is the most recent single-authored volume to appear. Fortunately, for readers and authors (and publishing companies), redundancy has been minimised, since each monograph vividly mirrors its author's unique interest, expertise, and personality.

Marchalonis devotes a full 10 chapters to a discussion of the evolution of immunoglobulin structure and function, an area in which his first-hand experience and insight is well-known. Following a detailed review of the structure of mammalian immunoglobulins and the arrangment of immunoglobulin genes in mammals, Marchalonis digests, in a well organised and well written fashion (and in separate chapters) the large body of literature dealing with invertebrate humoral mediators of defense; the phylogenetic emergence of IgM; light chains; the distribution of immunoglobulins distinct from the IgM class; variable regions; antigenic studies of immunoglobulins of eutherian mammals; molecules that might possibly be related to immunoglobulins; and the origins of antibody diversity. His text is more than a paraphrasing of the original papers; it is an assimilation that focuses on concepts and enigmatic problems as well as on facts.

Although Marchalonis does not deal with the evolution of the cellular basis of immunity with the same detail and perceptive depth that he devotes to humoral immunity, he does critically discuss many of the salient aspects of quasi-immune recognition and effector systems of invertebrates; the emergence of lymphoid cells and organs; the phylogenetic distribution of cellmediated immune functions; memory and tolerance; and manipulations of the immune systems of ectothermic vertebrates with temperature. It is in these chapters, however, that one shortcoming of the book emerges, a blemish that is revealed only because of the very vitality of the field with which it deals. Although fewer than $2 \%$ of the more than 700 cited references were published in 1975 (none were later), it is precisely during the past three years that many of the queries commendably raised by Marchalonis that bear, for example, on the evolution of lymphocyte heterogeneity, the evolution of the major histocompatibility complex, cell cooperation, and the selective effects of temperature on a subset of $T$ cells, have been approached and partly answered. It is also during this period that major concepts in cellular immunology have emerged that may have striking ramifications in understanding the evolution of immunity (for example, associated recognition). In short, the time from submission and revision of the manuscript to its publication this year was too long.

Fortunately, it is relatively easy for the interested reader to remedy this understandable problem. $\mathrm{He}$ or she need only peruse one of the recent photo-offset published proceedings of the symposia held in 1975-77 (Developmental Immunology, Am. Zool., 15, 3-213; 1975; Immunologic Phylogeny, ed. W. H. Hildemann and A. A. Benedict, Adv. exp. Med. Biol., 64, Plenum: New York; 1975; Phylogeny of Thymus and Bone-Marrow-Bursa Cells, ed. R. K. Wright and E. L. Cooper, North-Holland: Amsterdam; 1976; Developmental Immunobiology, ed. B. M. Solomon and J. D. Horton, North-Holland: Amsterdam; in press) to update their information. Marchalonis' book has provided many of the provocative questions and has predicted several experimental scenarios; these symposia provide at least some of the answers.

Nicholas Cohen is Associate Professor of Immunology at the University of Rochester School of Medicine and Dentistry, Rochester, New York.

\section{Liquid semiconductors}

Liquid Semiconductors. By Melvin Cutler. Pp. 226. (Academic: New York and London, 1977.) \$19.50; £13.85.

Physicisrs weaned on the nearly-freeelectron theory of solids find it satisfying, if at first surprising, that the properties of most liquid metals can be treated within this approximation. As long as the scattering of the conduction electrons by the non-crystalline arrangement of atoms is weak, perturbation theory can be used, as for example in Ziman's theory of electrical conductivity. It seems that many of the recently discovered metallic glasses can also be described in these terms. However, if the scattering is so strong that the mean free path becomes comparable to the average lattice spacing, then perturbation theory is no longer adequate and new approaches must be sought. This is the situation in certain liquid metals and in all amorphous and liquid semiconductors.

Concepts required for an understanding of the physics of amorphous and liquid semiconductors have evolved over the past ten years. It is clear that many properties are determined by the short range or local configuration of atoms - that is, bond length, bond angle and coordination number. These determine the main features of the distribution in energy of the electronic states. It is quite proper, therefore, that the author of this book should stress the tight-binding or molecular-bond model when interpreting experimental behaviour. Electrical conductivity, Hall effect and other transport properties demand special treatment, since the concept of well defined wave vectors for the electrons becomes inappropriate and the relaxation time approximation breaks down. The author describes the 